\title{
Análisis de la producción de conocimiento de los egresados de la especialización en salud de la familia del Programa Mais Médicos
}

\author{
Pedro Brito Quintana, ${ }^{1}$ Carlos Rosales, ${ }^{2}$ Gabriel Vivas Francesconi, ${ }^{3}$ Carolina Carvalho Novaes, ${ }^{2}$ \\ Viviane Paula Santos Rocha ${ }^{2}$ y Thiago Augusto Hernandes Rocha ${ }^{2}$
}

Forma de citar Quintana PB, Rosales C, Vivas Francesconi G, Novaes CC, Rocha VPS, Rocha TAH. Análisis de la producción de conocimiento de los egresados de la especialización en salud de la familia del Programa Mais Médicos. Rev Panam Salud Publica. 2020;44:e37. https://doi.org/10.26633/RPSP.2020.37

RESUMEN

Objetivo. Identificar las principales categorías de análisis en los trabajos de conclusión de curso de los médicos egresados de la especialización en Salud de la Familia del Programa Mais Médicos.

Métodos. Estudio de carácter bibliométrico de los 3021 trabajos de conclusión del curso disponibles en la base de datos del Acervo de Recursos Educativos en Salud, Universidad Abierta del Sistema Único de Salud, entre los años de 2016 y 2019. Para analizar los principales temas abordados se realizó un análisis por grupos, en dos etapas, de los descriptores referentes a cada trabajo.

Resultados. De los 3021 trabajos de conclusión de curso, se analizaron 2750 con datos completos. En cuanto al perfil de los autores, hay una predominancia del sexo femenino, origen cubano y grupo etario de 31 a 40 años. Los resultados también evidencian una concentración de los núcleos de formación de los profesionales, así como de algunos conceptos alineados a los preceptos trabajados con mayor frecuencia relacionados al objeto de la atención primaria de salud.

Conclusiones. El análisis del perfil de los trabajos de conclusión de curso destacó que los conceptos trabajados a lo largo del proceso de formación de los profesionales del Programa Mais Médicos se orientaron sobre todo a fortalecer la comprensión de las directrices estructurantes de la atención primaria. También logra el objetivo de acercar la capacitación médica sobre los problemas más relevantes en cada región del país.

Palabras clave Atención primaria de salud; educación continua; capacitación de recursos humanos en salud; Brasil.

La historia de la salud pública en Brasil reúne capítulos que revelan un largo camino hacia lo que hoy se conoce como sistema único de salud (SUS). Todos los puntos que se agregan a la historia de la salud pública en el país dejan claro el reto de proveer asistencia universal y llevarla hasta donde se halla la necesidad. Para eso, establecer políticas públicas de capacitación continua de profesionales, mejorar la distribución demográfica de médicos y monitorizar datos son desafíos que exigen estrategias de enfrentamiento capaces de evitar los prejuicios sobre la escasez de médicos, así como prevenir la inadecuada concentración o formación de estos $(1,2)$.

En Brasil, como en muchos países, es necesario discutir la demografía médica, dado que los médicos se siguen concentrando en las grandes ciudades y mantienen la desigualdad relacionada con la distribución de fuerza de trabajo en salud. Según la encuesta Demografía Médica en Brasil, el sudeste era

\footnotetext{
Organización Panamericana de la Salud/Organización Mundial de la Salud (OPS/OMS), Washington DC, Estados Unidos de América.

2 OPS/OMS, Brasilia, Brasil. $\square$ Thiago Augusto Hernandes Rocha, rochahernandes3@gmail.com
}

OPS/OMS, Nassau, Bahamas. 
la región con la mayor cantidad de médicos por cada 1000 habitantes $(2,67)$ contra 1,2 médicos en el noreste y 1,01 médicos en el norte $(3,4)$.

En este escenario, en julio de 2013, el gobierno de Brasil inició el Programa Mais Médicos (PMM) que se institucionalizó como política de estado mediante la Ley $\mathrm{N}^{\circ} 12871$ del 22 de octubre de ese año. El PMM, mediante la dotación de médicos para la totalidad del país, trató de mejorar la cobertura y la calidad de atención, tanto a nivel rural como urbano (5). El PMM presentaba tres ejes centrales: ampliación y mejora de la infraestructura de las unidades básicas de salud (UBS), formación para el SUS y provisión de emergencia de profesionales médicos. En relación con el eje de provisión de emergencia, formaron parte del PMM médicos de 62 nacionalidades, la mayoría cubanos, así como profesionales brasileros.

El PMM también tenía como finalidad estimular la integración enseñanza-servicio como fundamento para el cambio en la lógica de trabajo y de formación en salud $(6,7)$.

En este sentido, un aspecto diferencial del PMM es la incorporación obligatoria de los médicos en actividades de formación complementaria, con propuestas metodológicas dirigidas a temas que mejoran la práctica de la medicina de familia y comunidad. Esos programas deberían calificar al profesional médico en la perspectiva del modelo y de las políticas de salud brasileña $(2,8)$. En esas políticas, la atención primaria de salud (APS) aparece como escenario estratégico para la reorientación de la formación de los profesionales de salud y aproximarlos a las necesidades de la población determinadas tanto desde el punto de vista social como histórico. Además de esos aspectos, permite más posibilidades de desarrollo de la autonomía de los individuos en la definición de los actos en salud más coherentes con esas necesidades (9).

Las actividades de formación complementaria del PMM se distribuyen en dos ciclos formativos. El primero es el curso de especialización en salud de la familia, conducido por una institución de enseñanza superior cooperante del Sistema de la Universidad Abierta del SUS (UNASUS). El segundo ciclo busca profundizar el conocimiento del médico participante en temas relevantes relacionados con la APS, con actividades educativas en el nivel de perfeccionamiento y extensión (9).

A lo largo del cumplimiento de los ejes curriculares, se proponen actividades de reflexión para la elaboración de un proyecto de intervención, desarrollado como trabajo de conclusión del curso (TCC). Los trabajos presentados por los alumnos participantes del PMM son proyectos de intervención locales, que consisten en una propuesta de acción y que deberían tener como foco la mejora de las condiciones de salud de la población en el contexto de la APS.

La magnitud del PMM añadida a su carácter inédito ha inspirado muchos estudios, entre los cuales podemos mencionar las pesquisas de opinión sobre el PMM, evaluaciones del impacto sobre los servicios de salud y la valuación de los programas educacionales. Aun así, no se habían reunido, hasta el momento, las informaciones relacionadas a la producción académica de los egresados del curso de especialización por el cual pasaron todos los médicos participantes del programa. Así, este manuscrito presenta un estudio bibliométrico de los principales temas tratados en los TCC de los médicos egresados del PMM. El propósito de este trabajo fue identificar las principales categorías de análisis trabajadas en conjunto en los TCC.

\section{MATERIALES Y MÉTODOS}

Para este estudio, de carácter bibliométrico, se utilizaron aplicaciones de técnicas estadísticas para describir aspectos de producción y diseminación de conocimiento científico (10). La elección del método bibliométrico ayuda a la comprensión de temáticas y áreas nuevas, y contribuye así a la identificación de tendencias en la producción de conocimiento (10).

Se utilizaron datos de los trabajos de conclusión del curso, del Acervo de Recursos Educativos en Salud (ARES), el repositorio educativo de UNASUS. En él se ofrecen materiales sobre temáticas diversas del área de la salud, producidos por las instituciones de enseñanza superior que forman la Red UNASUS para la provisión de educación a distancia (11).

El análisis se concentró en los trabajos disponibles hasta marzo de 2019 que contaban con autorización de los autores para su publicación en la base ARES. Es decir, no se examinaron todos los trabajos producidos, sino aquellos que cumplen requisitos institucionales para ser publicados.

Con base en los trabajos de ARES, fue posible realizar un análisis orientado hacia una dimensión cuantitativa, considerando variables demográficas e institucionales de los participantes, y una dimensión cualitativa, que se centra en la identificación de las áreas temáticas de intervención del TCC.

El total de TCC en la plataforma ARES fue de 3021, incluidos el PMM y el Programa de Valorización del Profesional de la Atención Básica (PROVAB), publicados entre junio de 2016 y marzo de 2019. El universo de análisis fue definido en un total de 2750 TCC de especialización en salud de la familia elaborados solo por participantes del PMM. No se analizaron trabajos vinculados al PROVAB. Ante las variables disponibles en la base ARES, se identificaron cuáles serían utilizadas para la caracterización sociodemográfica de los autores (edad, género, nacionalidad, universidad de apoyo) y los Descriptores en Ciencias de la Salud (DeCS). El DeCS fue creado para servir como un lenguaje único para la indexación de contenido en el área de salud. Su uso posibilitó la uniformidad de los términos de indexación y la recuperación de la información (12).

Se reportaron 4877 áreas temáticas en 12 categorías para definir el tema de concentración del TCC. Para identificar qué conceptos se abordaron en cada área, fue necesario el análisis complementario con los DeCS. Como cada trabajo tenía diversos descriptores, los DeCS de cada TCC fueron sometidos a un análisis por grupos en dos etapas para identificar áreas temáticas en salud que se trabajaban de manera simultánea.

El análisis por grupos en dos etapas difiere de los análisis de conglomerados que suelen realizarse, pues permite que se consideren variables ordinarias y continuas de manera simultánea y con estructura multivariada, para definir agrupaciones de pertenencia (13). Los enfoques de agrupamiento más conservadores no se ajustan bien con grandes volúmenes de datos (14). El algoritmo de agrupamiento en dos etapas fue adaptado para el manejo de grandes volúmenes de datos, lo que permitió superar las dificultades asociadas a los análisis por grupos tradicionales (15). La categorización como de etapa doble se debe a la forma en que se analizan los datos para la definición de los conglomerados. Primero, hay una fase de agrupamiento inicial de las observaciones en subconglomerados. Después, cada subconglomerado es tratado como una unidad de análisis. Las decisiones sobre la migración de observaciones de un subconglomerado a otro se basan en criterios de distancia (16). 
El modelo de dos etapas permite la definición automática de conglomerados según los criterios de información bayesiano (BIC, por sus siglas en inglés) y de información Akaike (AIC, por sus siglas en inglés). La adecuación del número de conglomerados definidos es aún comprobada, en función del descenso en el logaritmo de la función de máxima verosimilitud (13). Esta función genera una estadística de calidad de agrupación llamada medida de silueta de cohesión y separación. Una buena silueta señala un resultado de buena calidad; es decir, grupos homogéneos en su interior y diferentes entre sí. Además, el modelo de agrupamiento en dos etapas es robusto para las cuestiones infracción de los supuestos de análisis, lo que garantiza estabilidad de resultados (15).

Para el análisis por grupos, se analizaron solo 2636 del total de 2750 TCC de egresados del PMM, en función de la existencia de informaciones en cuanto a los DeCS. Así, se consideraron en total 446 descriptores extraídos de esos 2636 TCC. Como cada TCC podría contener más de un descriptor, en total se analizaron 9556 ocurrencias de descriptores, de los cuales 199 aparecían solo una vez. Como parámetro del análisis se eligió el límite máximo de 10 conglomerados, en los cuales los descriptores se repitieron por lo menos 25 veces.

\section{RESULTADOS}

En la figura 1 se observa la distribución de los autores según edad y sexo de los 2750 TCC analizados. Se observa que la mayoría son mujeres $(1537,55,8 \%$ ) y la mayor concentración (1107) está en el grupo de edad de 31 a 40 años. El PMM, como es sabido, se compuso no solo de médicos cubanos $(1848,67,2 \%)$, sino también de brasileros $(563,20,5 \%)$ y otros extranjeros. Con respecto a los vínculos institucionales de los autores, la mayoría

FIGURA 1. Caracterización demográfica de los autores de los trabajos analizados, Brasil (2016-2019)

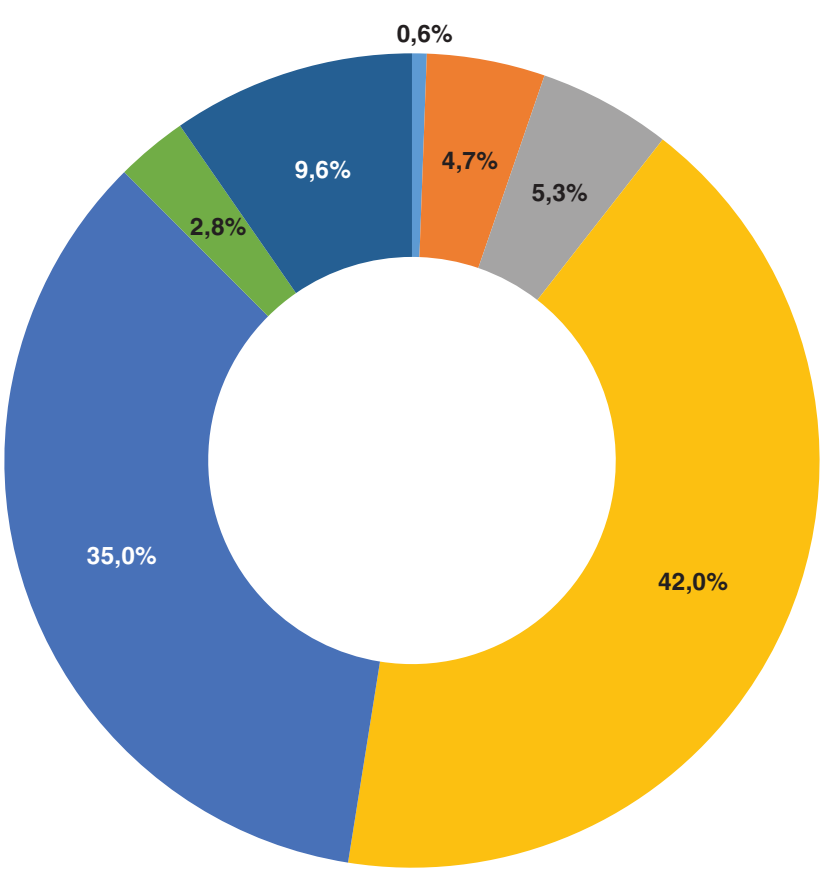

A. Universidad de origen
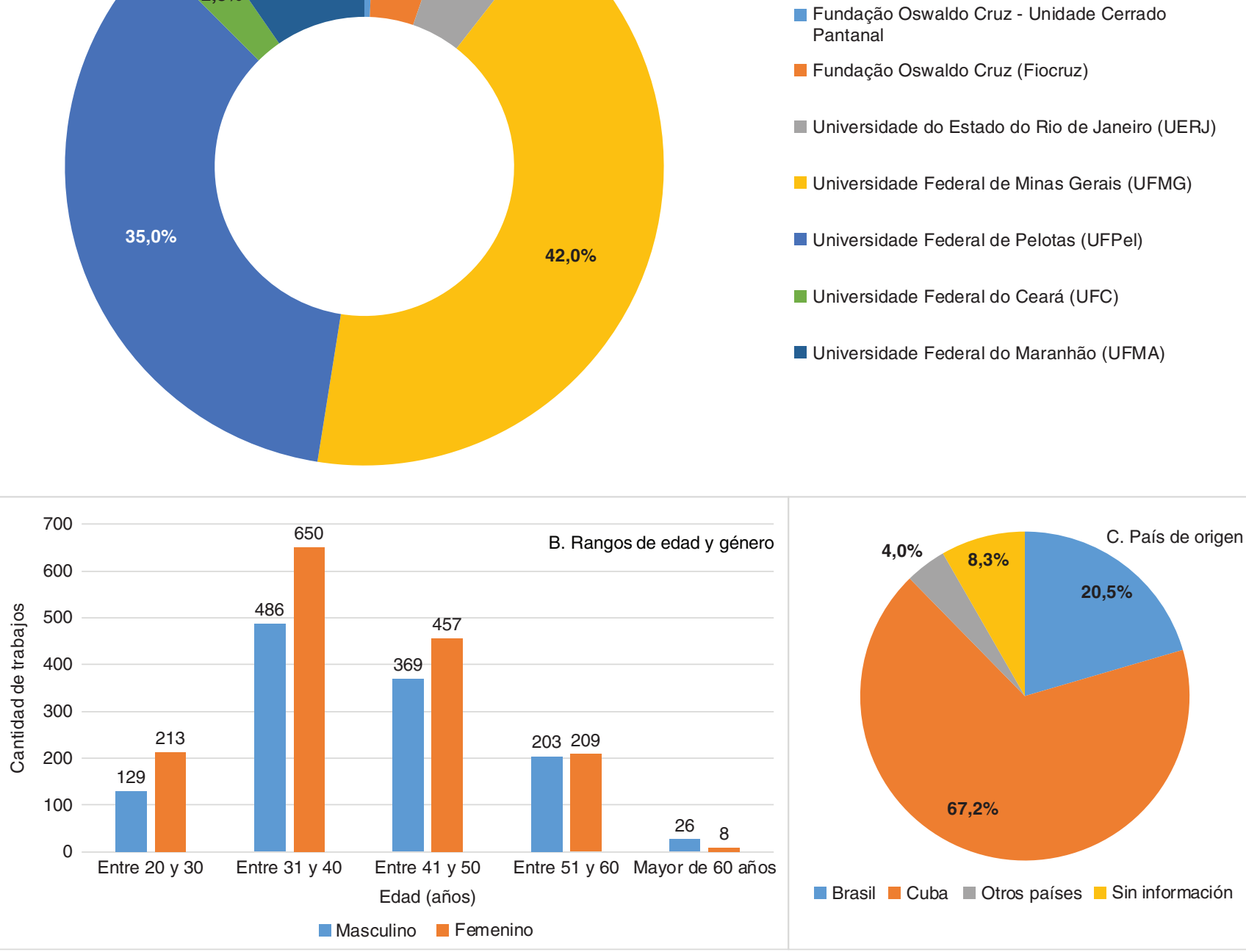


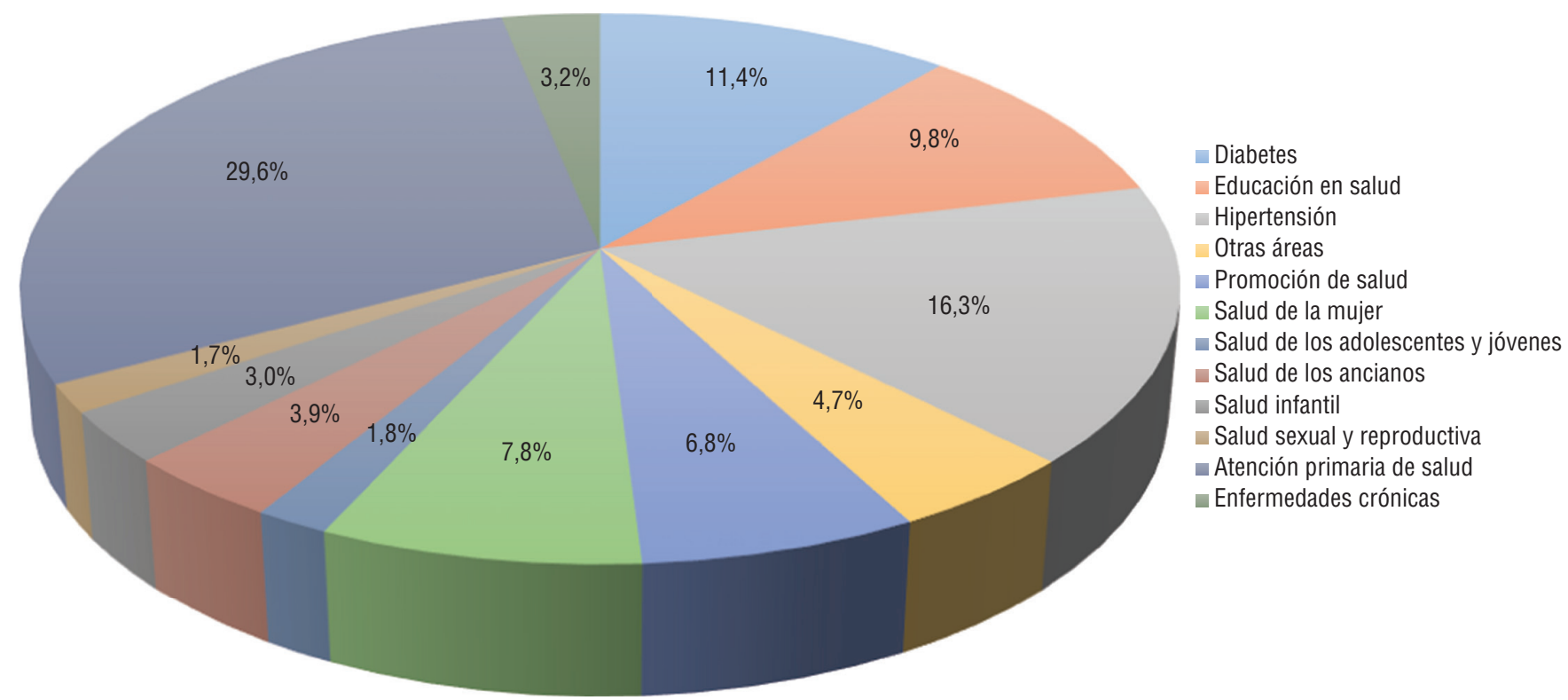

proviene de la Universidad Federal de Minas Gerais y de la Universidad Federal de Pelotas: $1154(42,0 \%)$ y 963 (35,0\%), respectivamente.

En relación con las áreas temáticas discutidas en los TCC, en a figura 2 se observa que la atención primaria en salud reúne $1444(29,6 \%)$ del total. Por otra parte, $794(16,3 \%)$ de los trabajos se dedicaron al tema de hipertensión, y $558(11,4 \%)$ a diabetes. Estos tres temas suman más de la mitad del total de TCC analizados 2796 (57,3\%). La distribución de los DeCS indicó que muchos de ellos ocurren pocas veces. Cerca de diez descriptores suman casi $80 \%$ de los conceptos trabajados en los documentos investigados (figura 3). En el análisis por grupos, el indicador de métrica de silueta de cohesión y separación fue clasificado como bueno, lo que indica que el agrupamiento propuesto consiguió aglutinar individuos semejantes en grupos homogéneos en su interior y entre grupos con máxima heterogeneidad entre sí. El conglomerado de menor tamaño presentó una agrupación de 673 descriptores, mientras que el más grande aglutinó 1308. La distribución de la proporción de DeCS entre los grupos varió desde $7,0 \%$ hasta un máximo de 13,7\%, lo que evidencia una distribución equilibrada de descriptores.

El análisis por grupos permitió identificar patrones de los descriptores abordados por TCC. Había 446 descriptores únicos para hacer agrupación, por lo que en algunos grupos se observó un peso mayor en la categoría "Otros". Esto refleja la ocurrencia de diversos descriptores con frecuencia baja dentro del conglomerado en cuestión. Las ventajas de utilizar el análisis por grupos reposan en la demostración de tendencias dominantes. En ese sentido, los demás descriptores no insertados junto a la categoría "Otros" son aquellos que marcan las principales tendencias, en términos de los conceptos trabajados junto a los TCC.

El primer conglomerado destacado en la figura 4 resalta que hay un conjunto de trabajos que abordaron el tema de la hipertensión arterial sistémica. En el segundo conglomerado, los temas cubiertos fueron la atención a la salud mental y la neoplasia de mama, junto a temas como programas de rastreo, atención domiciliaria y adherencia a la medicación. El conglomerado 3 fue marcado por el debate sobre temas vinculados con la obesidad y neoplasias del cuello uterino. El cuarto conglomerado se concentró en trabajos que vincularon sobre todo temas de salud infantil y salud oral. El quinto conglomerado reúne de manera casi exclusiva a los trabajos que analizaron elementos de la atención primaria de salud. El sexto trató sobre enfermedades crónicas vinculadas con temas diversos.

En la figura 5 se observa que el séptimo conglomerado abordó temas diversos, sin una dominación de descriptores específicos. El octavo vinculó cuestiones de salud de la mujer y adultos mayores. Los dos últimos conglomerados abordaron descriptores vinculados a la diabetes mellitus y a la salud de la familia, respectivamente.

Los resultados evidencian una concentración en lo que se refiere a los núcleos de formación de los profesionales en cuestión, así como la predominancia de algunos conceptos alineados a los preceptos trabajados con mayor frecuencia, junto al objeto de la atención primaria. Algunas categorías demostraron predominancia en cuanto a las áreas temáticas de los trabajos producidos. Los conglomerados no siguieron necesariamente los mismos patrones demostrados en las áreas temáticas, pues el análisis por grupos examina qué grupos de descriptores ocurren de forma conjunta.

\section{DISCUSIÓN}

A partir de la identificación de las principales categorías de análisis en los TCC de los médicos del PMM fue posible caracterizar los principales temas planteados en los trabajos de la especialización en salud de familia.

Sobre los primeros resultados presentados, se comprende que el gran énfasis dado a la atención primaria y las enfermedades crónicas no transmisibles es consistente con el enfoque dado a estos temas en las propuestas de las instituciones educativas y 
FIGURA 3. Distribución de los descriptores y calidad de los conglomerados, Brasil (2016-2019)

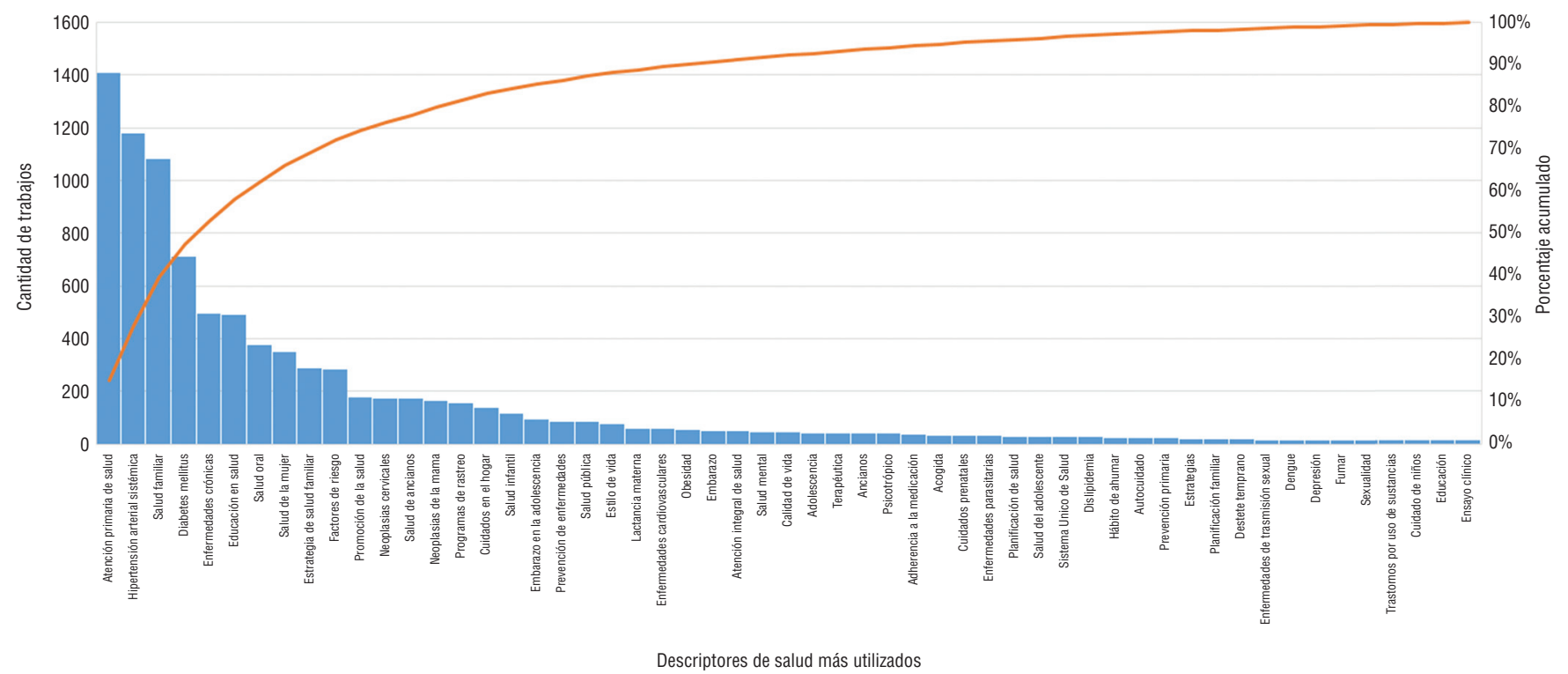

Tamaños de conglomerados
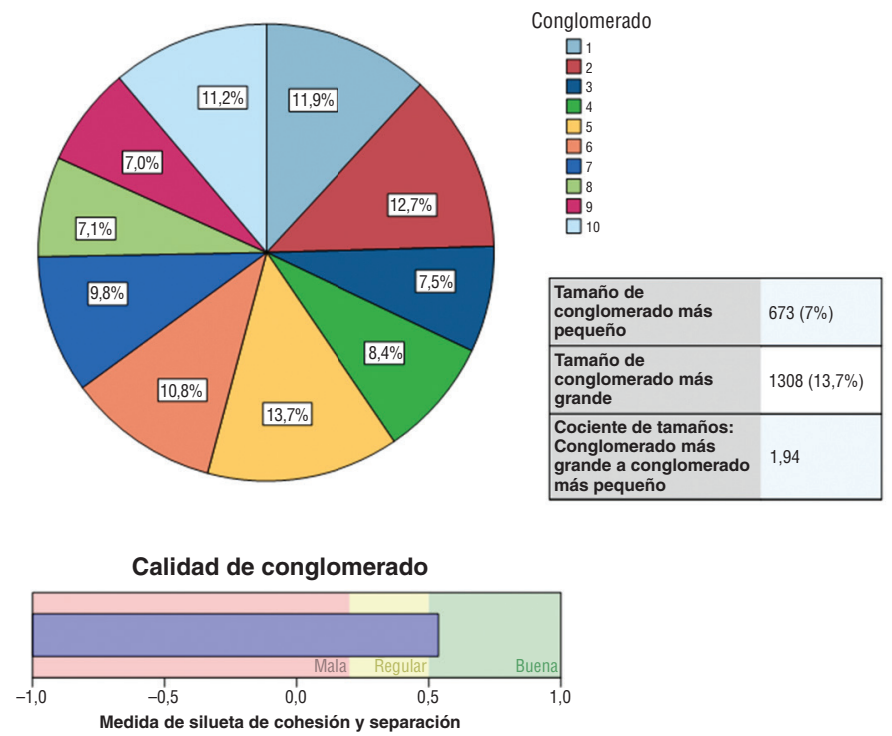

con los datos de morbilidad y mortalidad en Brasil. El curso de especialización viabilizó la calificación de los profesionales participantes del Programa Mais Médicos. Las actividades educativas del curso aportaran el desarrollo de conocimientos y habilidades basadas en las circunstancias del territorio de trabajo de los médicos. De esta manera, contribuyen al reconocimiento, identificación y análisis de indicadores epidemiológicos de su área de actuación.

El análisis de los trabajos de conclusión de curso, a pesar de incapaz, por sí solo, de evidenciar un cambio en la praxis médica, destacó que los conceptos trabajados durante el proceso de formación de los profesionales del Programa Mais Médicos se dirigieron al fortalecimiento del entendimiento de las directrices que estructuran la atención primaria.
Los trabajos producidos por los médicos del Proyecto Mais Médicos fueron apoyados por tres universidades importantes del país, en términos de calidad de enseñanza. El hecho de que las instituciones estén ubicadas em diferentes regiones ha contribuido para que la mirada hacia los retos en el campo de la atención básica sea diversificada y más rica, principalmente cuando se tiene en cuenta la dimensión geográfica y cultural del país (17). El perfil de los conglomerados identificados indicó un predominio de temas que abordaron cuestiones centrales para la atención primaria, tales como enfermedades crónicas, atención domiciliaria, salud familiar, prevención de neoplasias, salud infantil y salud de los adultos mayores. Se abordaron otros temas, pero sin que fueran determinantes para definir un conjunto de conceptos trabajados siempre de forma unificada. 
FIGURA 4. Programa Mais Médicos: categorías principales cubiertas en los conglomerados 1-6 de los DeCS, Brasil (2016-2019)
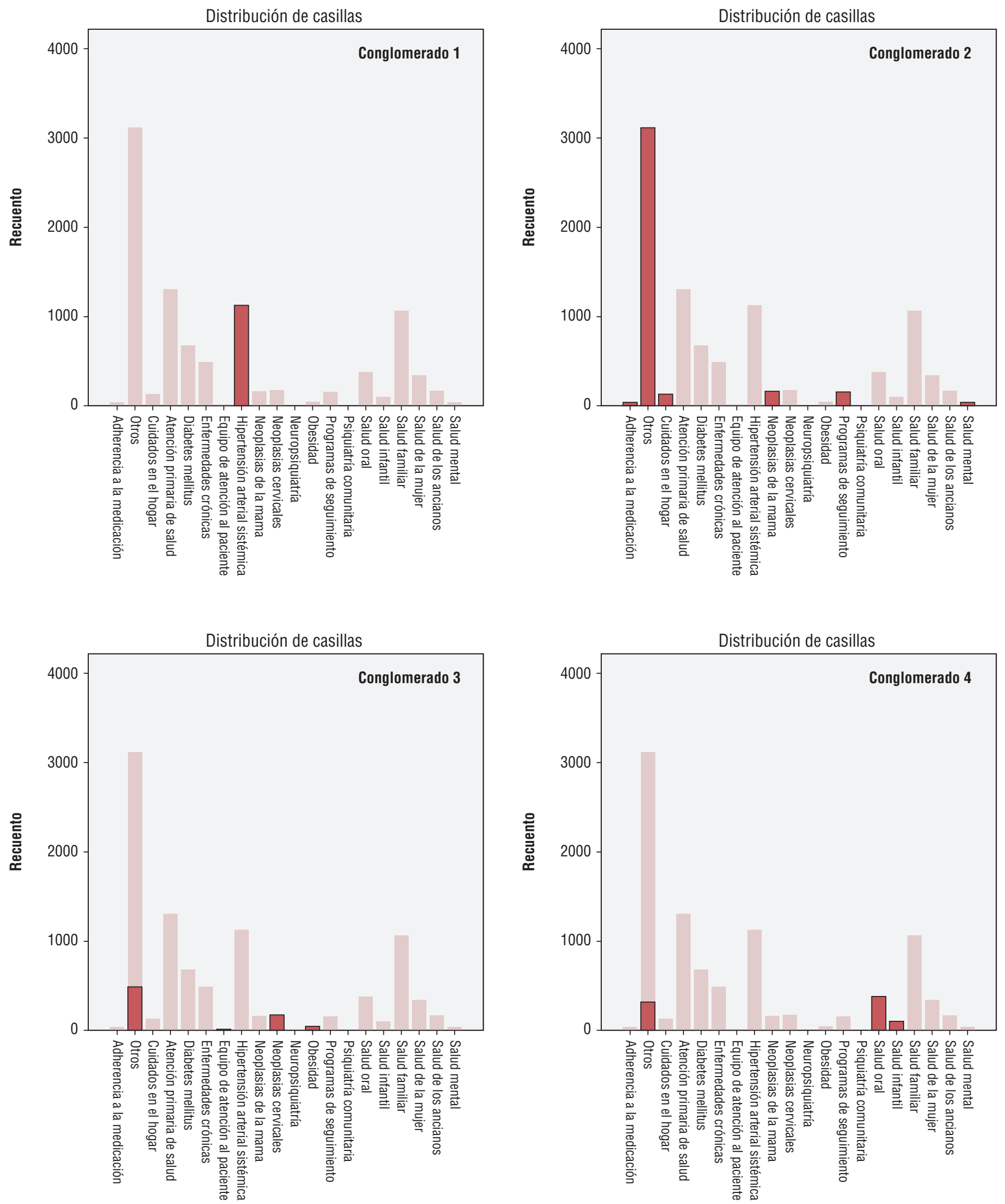


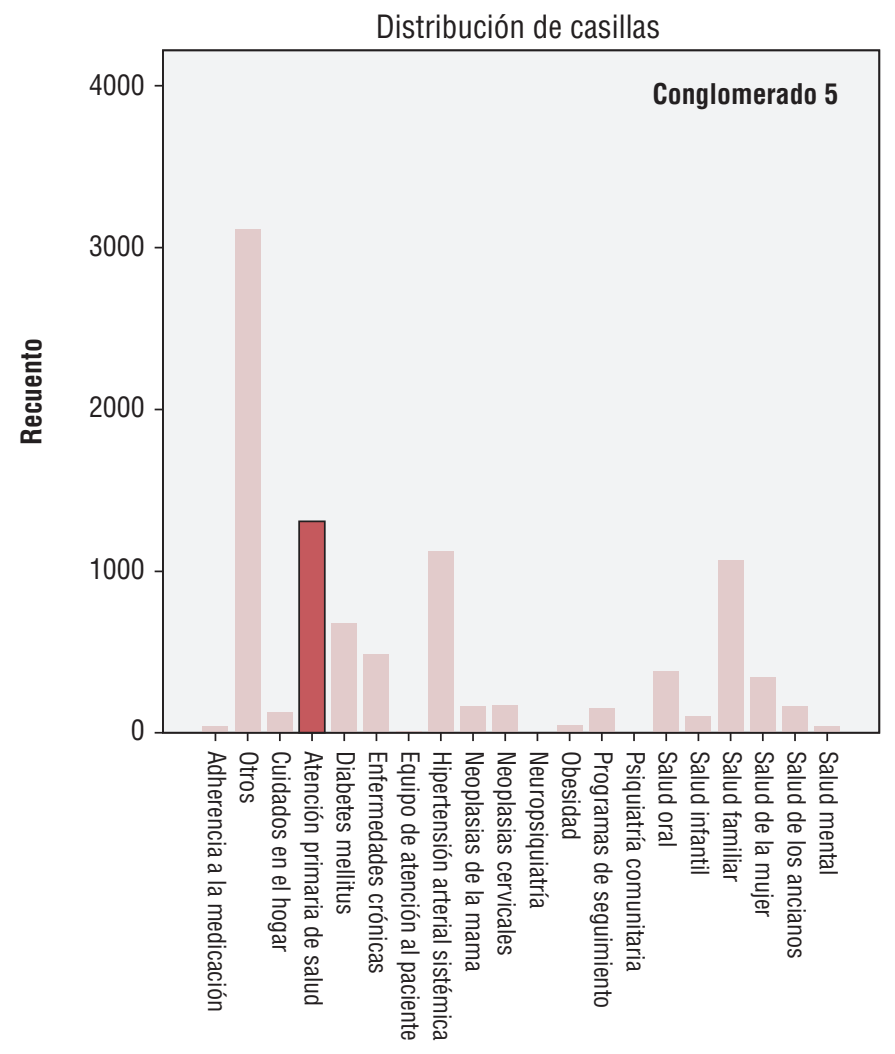

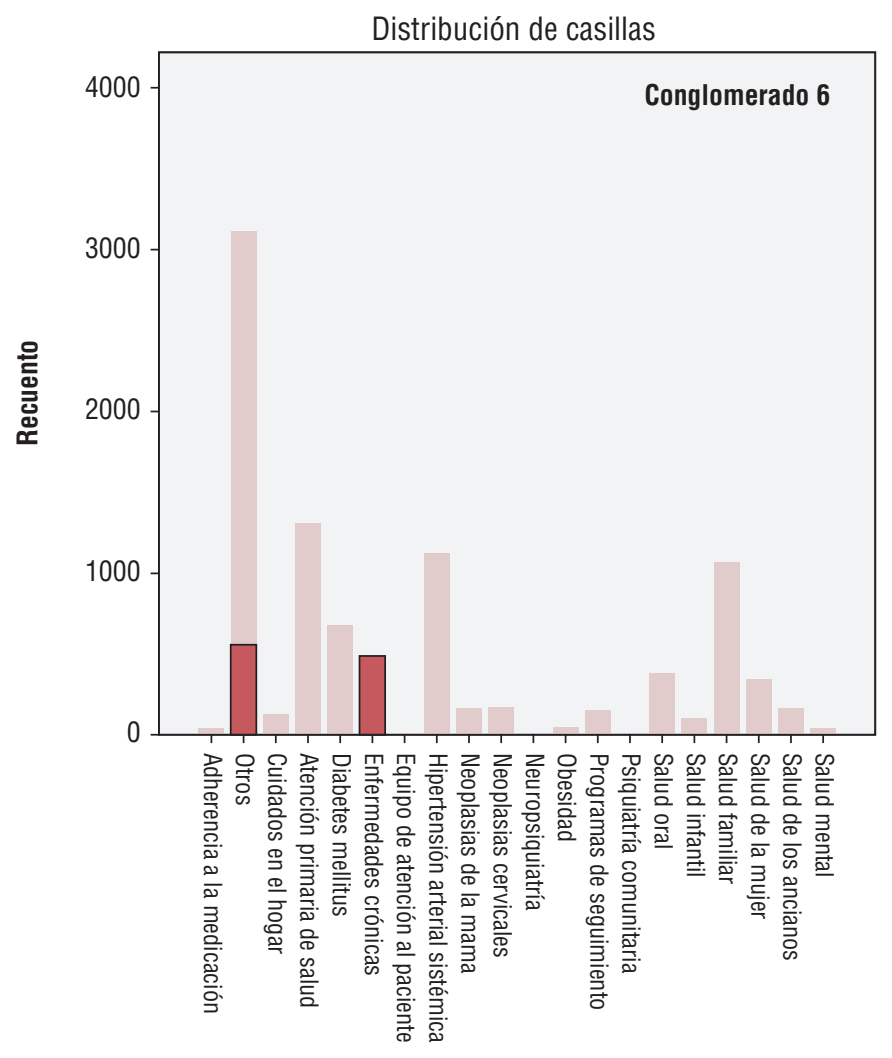

Suma de trabajos que citaron la categoría en cuestión para el conglomerado analizado
Es decir, el bloque de trabajos producido constituye un saber aplicado a salud y, por lo tanto, puede ofrecer a los profesionales una gama de opciones prácticas para la mejoría del trabajo ejercido. Esto es aun más evidente, si se agrega el hecho de que estas prácticas se distribuyen por el país y que cada profesional tuvo que mirar hacia su realidad para proponer una acción que haga frente a los problemas que identificó. Esto se demuestra, por ejemplo, por la concentración de temas en el conglomerado 5 , que reúne temas relacionados con la atención primaria cualquiera sea la ubicación geográfica.

El debate de temas relacionados con los preceptos fundamentales de la atención básica, tales como prevención y promoción de enfermedades crónicas, la diversidad de grupos poblacionales prioritarios es evidente en las concentraciones de los conglomerados 2, 3, 4 y 8. En ellos se pone de manifiesto que el proceso de desarrollo de los TCC buscó, en alguna medida, cuestiones de interés para la formación de profesionales especializados en cuidados determinados por la prevención primaria de enfermedades.

El PMM ofrece a los médicos que forman parte de él actividades de educación continua que involucran enseñanza, investigación y extensión bajo la orientación de médicos supervisores y tutores, en el contexto de la especialización que brinda el SUS (8). La actuación del médico del PMM en los territorios busca fortalecer y expandir la capacidad de intervención y resolución de problemas a través de la actuación integrada con los demás profesionales que componen los equipos de atención primaria de salud (9). En este sentido, las actividades de formación constituyen la oportunidad para el fortalecimiento de la capacidad de desarrollo de competencias necesarias para el cambio del modelo de atención a la salud, entre ellas, las relacionadas a la formación de sujetos más aptos para el trabajo en equipo $(6,17,18)$.

Como limitación del presente estudio, cabe destacar la imposibilidad de analizar el total de los trabajos de los médicos egresados del proceso de formación, ya que no todos permitieron la divulgación del resultado de sus respectivos trabajos de conclusión de curso en la base de datos de ARES. Además, no todas las universidades responsables del proceso de capacitación de médicos han tenido una estructura de repositorio de documentos organizada y con acceso público para permitir el examen de los trabajos de conclusión de curso.

En lo que se refiere a beneficios de gestión, se recomienda que haya devolución de los datos ordenados y sistematizados a los servicios para que tengan en cuenta el conocimiento que ayudaron a producir y cómo se benefician de la aplicación completa o parcial de las acciones. La estrategia de combinar acciones de capacitación para profesionales médicos involucrados en los servicios de atención primaria permitió el desarrollo de estudios teóricos y aplicados centrados en los principales 
FIGURA 5. Programa Mais Médicos: categorías principales cubiertas en los conglomerados 7-10 de los DeCS, Brasil (2016-2019)
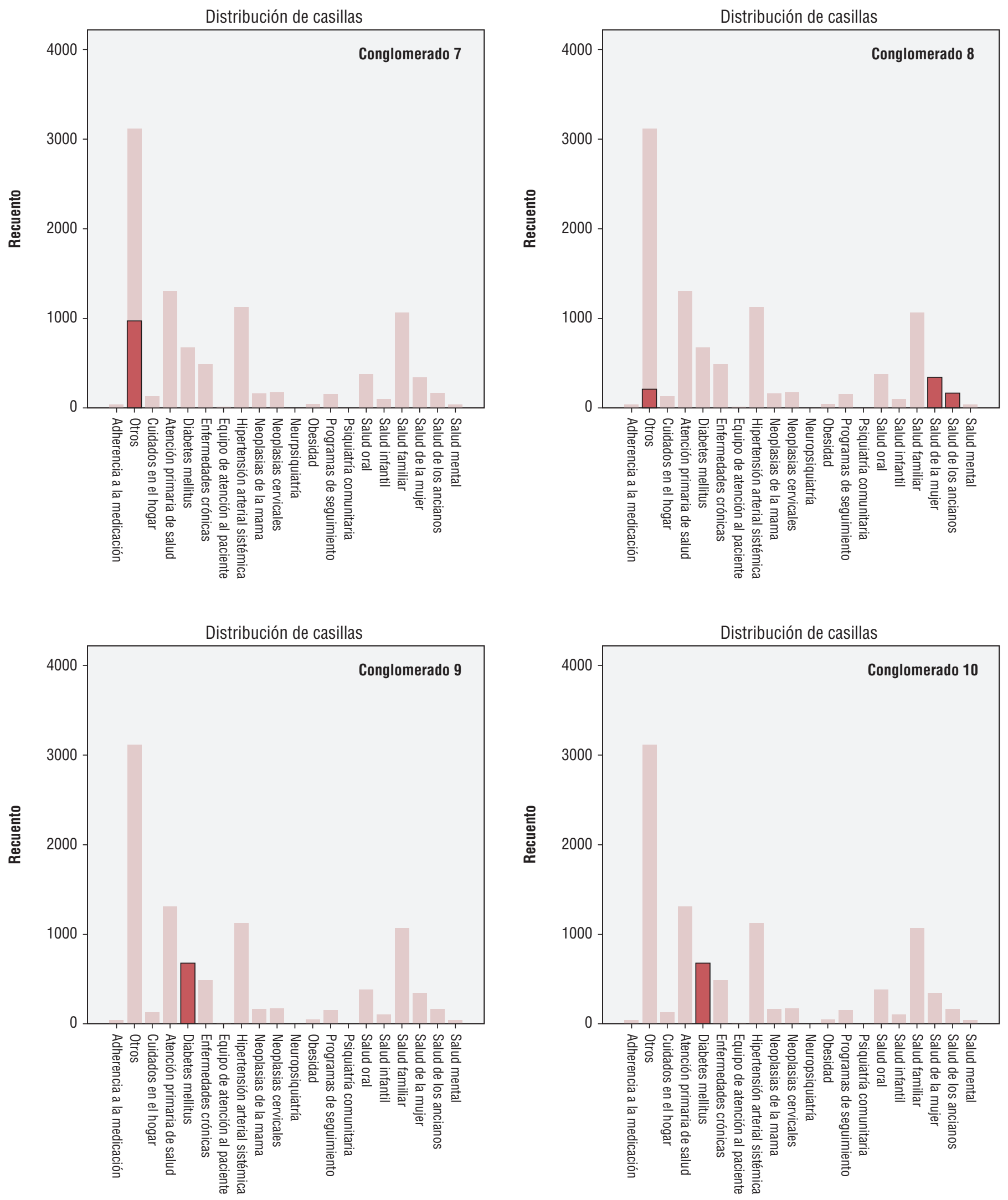
temas relacionados con la atención primaria de salud. En cuanto al avance de conocimiento, se sugiere que se realicen estudios que investiguen la relación entre los temas más frecuentes y la epidemiología brasilera que seleccionen las propuestas más consistentes para pilotos y confirmaciones de su validez. Con una mirada más amplia, identificar las categorías de los temas de trabajos realizados por egresados de la especialización en Brasil tiene gran valor para el fortalecimiento de temas de origen pragmática dentro del alcance de la atención primaria de salud.
Contribución de los autores. Todos los autores contribuyeron de igual manera en la edición, revisión, análisis y escritura del manuscrito.

Conflicto de intereses. Ninguno declarado por los autores.

Declaración. Las opiniones expresadas en este manuscrito son responsabilidad del autor y no reflejan necesariamente los criterios ni la política de la RPSP/PAJPH y/o de la OPS.

\section{REFERENCIAS}

1. Mourão Netto JJ, Rodrigues AR, Aragão O, Goyanna N, Cavalcante AE, Vasconcelos MA, et al. Programa Mais Médicos e suas contribuições para a saúde no Brasil: revisão integrativa. Rev Panam Salud Publica. 2018;42:1-7. Disponible en: http://iris.paho.org/ xmlui/handle/123456789/34893

2. Amaral PV, Francesconi GV, Rosales C, Kemper ES, da Silva NC, Soares JGN, et al. Programa Mais Médicos: como avaliar o impacto de uma abordagem inovadora para superação de iniquidades em recursos humanos. Rev Panam Salud Publica. 2018;42:e185. Disponible en: http:/ /iris.paho.org/xmlui/handle/123456789/49564

3. Scheffer M et al. Demografia médica no Brasil 2015. Departamento de Medicina Preventiva, Faculdade de Medicina da USP. Conselho Regional de Medicina do Estado de São Paulo. Conselho Federal de Medicina; 2015.

4. Scheffer M et al. Demografia médica no Brasil. Departamento de Medicina Preventiva, Faculdade de Medicina da USP. Conselho Regional de Medicina do Estado de São Paulo. Conselho Federal de Medicina; 2018.

5. Brasil. Lei No 12.871, de 22 de outubro de 2013 - Institui o Programa Mais Médicos. Brasília; 2013.

6. Santos LMP, Oliveira A, Trindade JS, Barreto IC, Palmeira PA, Comes $Y$, et al. Implementation research: towards universal health coverage with more doctors in Brazil. Bull World Health Organ. 2017;95(2):103-12.

7. Santos LMP, Costa AM, Girardi SN. Programa Mais Médicos: uma ação efetiva para reduzir iniquidades em saúde. Cien Saude Colet. 2015;20(11):3547-52. Disponible en: http://www.scielo.br/ scielo.php?script=sci_arttext\&pid=S1413-81232015001103547\&lng= pt\&tlng $=$ pt

8. Brasil. Ministério da Saúde. Secretaria de Gestão do Trabalho e da Educação na Saúde. Programa mais médicos - dois anos: mais saúde para os brasileiros. Brasilia; 2015.

9. Freire Filho JR, Magnago C, Costa MV da, Forster AC. Cursos de especialização ofertados no âmbito do Mais Médicos: análise documental na perspectiva da educação interprofissional. InterfaceComun Saúde; 2018.

10. Wallin JA. Bibliometric methods: pitfalls and possibilities. Basic Clin Pharmacol Toxicol. 2005;97(5):261-75. Disponible en: http:// www.ncbi.nlm.nih.gov/pubmed/16236137
11. Oliveira V de A, Savassi LCM, Lemos AF, Campos FE de. eLearning for health in Brazil - UNA-SUS in Numbers. J Int Soc Telemed EHealth. 2013;4:e9:1-7. Disponible en: https://journals.ukzn.ac.za/ index.php/JISfTeH/article/view/139

12. Biblioteca Virtual en Salud Descriptores en ciencias de la salud. DeCS Server - List Terms. OMS/OPS; 2018.

13. Costa, Oliveira GG. Uso da técnica two step cluster para segmentação de funcionários de uma empresa no Rio de Janeiro segundo clima organizacional: um estudo de caso. Rev da Estatística da Univ Fed Ouro Preto. 2017;6(1). Disponible en: http://www.cead.ufop. $\mathrm{br} /$ jornal/index.php/rest/article/view/706

14. Kaufman L, Rousseeuw PJ. Finding groups in data: an introduction to cluster analysis (Wiley Series in Probability and Statistics). Eepe Ethz Ch; 1990.

15. Tkaczynski A. Segmentation using two-step cluster analysis. En: Segmentation in social marketing: process, methods and application. Springer; 2016.

16. Tevdovski D, Trpkova M. Twostep cluster analysis: segmentation of largest companies in Macedonia. En: Kovács P, Szep K, Katona T, editors. Challenges for analysis of the economy, the businesses, and social progress. Szeged: Universitas Szeged Press; 2009:302-20.

17. Girardi SN, Carvalho CL, Pierantoni CR, Costa J de O, Stralen AC de $S$ van, Lauar TV, et al. Avaliação do escopo de prática de médicos participantes do Programa Mais Médicos e fatores associados. Cien Saude Colet. 2016;21(9):2739-48.

18. Santos LMP, Costa AM, Girardi SN. Programa Mais Médicos: uma ação efetiva para reduzir iniquidades em saúde. Cien Saude Colet. 2015;20(11):3547-52. Disponible en: http://www.scielo.br/scielo. php?script=sci_arttext\&pid=S1413-81232015001103547\&lng=pt\&n$\mathrm{rm}=$ iso\&tlng=en

Manuscrito recibido el 20 de mayo de 2019. Aceptado para su publicación, tras revisión, el 25 de setiembre de 2019. 


\section{Analysis of knowledge production by graduates of the family health specialization in the Mais Médicos Program}

ABSTRACT Objective. Identify the main categories of analysis in the end-of-course projects completed by physicians who graduated in the family health specialization in the Mais Médicos Program.

Methods. Bibliometric study of the 3,021 end-of-course projects found in the database of the Health Education Resources Collection (Open University of the Unified Health System) for the years 2016 to 2019. In order to analyze the main topics addressed in the projects, a two-step cluster analysis was conducted of the descriptors for each project.

Results. Of the 3,021 end-of-course projects, 2,750 with complete data were analyzed. The profile of the authors was predominantly female, Cuban, and in the 31-40 years age group. The results show that professional training was concentrated in specific topics, most of them closely related to the goals of primary health care.

Conclusions. The analysis of the profile of the end-of-course projects showed that the topics studied throughout the process of training the professionals in the Mais Médicos Program were focused mainly on greater understanding of the structural directives of primary care. Furthermore, the objective of focusing medical training on the most relevant problems in each region of the country was achieved.

Keywords $\quad$ Primary health care; education, continuing; health human resource training; Brazil.

\section{Análise da produção de conhecimento por egressos da especialização em saúde da família no Programa Mais Médicos}

RESUMO

Objetivo. Identificar as principais categorias de análise nos trabalhos de conclusão de curso realizados por médicos formados na especialização em saúde da família do Programa Mais Médicos (PMM).

Métodos. Realizou-se um estudo bibliométrico que incluiu 3021 trabalhos de conclusão identificados na base de dados da Coleção de Recursos Educativos em Saúde da Universidade Aberta do Sistema Único de Saúde (UMA-SUS) para os anos de 2016 a 2019. Os principais tópicos abordados nos projetos foram estudados a partir da análise em duas etapas dos descritores.

Resultados. Dos 3021 trabalhos de conclusão, $2750 \mathrm{com}$ dados completos foram analisados. Os autores foram predominantemente do sexo feminino, cubanos e na faixa etária de 31 a 40 anos. Os resultados mostram uma concentração em temas específicos, a maioria intimamente relacionada com os objetivos da atenção primária à saúde.

Conclusões. A análise do perfil dos trabalhos de conclusão mostrou que os temas estudados ao longo do processo de formação dos profissionais do PMM enfocaram principalmente a maior compreensão das diretrizes estruturais da atenção primária. Além disso, foi alcançado o objetivo de direcionar a formação médica aos problemas mais relevantes em cada região do país.

Palavras-chave Atenção primária à saúde; educação continuada; capacitação de recursos humanos em saúde; Brasil 